\title{
THE CELL-LIKE MAPPING PROBLEM
}

\author{
BY GEORGE KOZLOWSKI AND JOHN J. WALSH ${ }^{1}$
}

A major unresolved issue in topology is whether or not there is a dimension raising cell-like mapping. A cell-like map $f: X \rightarrow Y$ is a proper mapping between metrizable spaces such that $f^{-1}(y)$ has the shape of a point for each $y$ $\in Y$ (i.e., every map of $f^{-1}(y)$ to a polyhedron is null-homotopic or, equivalently for finite dimensional $X, f^{-1}(y)$ admits a cellular embedding in a Euclidean space).

During the period of his pioneering work on cellular decompositions of 3space, Bing asked if the quotient space of such a decomposition is an ANR [Bi]. In the mid-sixties, Armentrout asked if the quotient space of a cellular decomposition of $E^{3}$ is finite dimensional [Ar]. It was shown in [Koz-1] that these two questions are equivalent and both are settled affirmatively by the following theorem.

THEOREM. If $f: X \rightarrow Y$ is a cell-like mapping defined on a subset of a 3-manifold, then $\operatorname{dim} Y \leqslant 3$.

The theorem is best understood by considering the case with $X$ a compact subset of $E^{3}$. The Sphere Theorem easily implies that (1) a cell-like subset of $E^{3}$ has arbitrarily small aspherical open neighborhoods and (2) each component of the intersection of two aspherical open subsets of $E^{\mathbf{3}}$ is aspherical. In [Koz-2], the cell-like mapping problem is reduced to an extension problem for maps into polyhedra; this extension problem is solvable for maps into aspherical polyhedra. These facts are combined to prove the theorem by producing an $\epsilon$-map of $Y$ into $E^{3}$ for each $\epsilon>0$.

It was known previously that cell-like maps on 1-dimensional spaces do not raise dimension. This is proved by using the Vietoris Mappirg Theorem to conclude that the image has (integral) cohomological dimension at most 1 and then appealing to the fact that covering dimension agrees with cohomological dimension in this case (both are "classified" by extensions of maps into $S^{\mathbf{1}}$ ). The precise connection between the cell-like mapping problem and the classical question of whether or not covering dimension and cohomological dimension agree for compacta was recently established by R. D. Edwards [Ed-1]; he announced that a compactum with cohomological dimension $\leqslant n$ is the cell-like image of a compactum with covering dimension $\leqslant n$.

It seems appropriate to sketch two reductions of the cell-like mapping problem for such maps on ANR's and manifolds. First, the result of Bothe [Bo]

Received by the editors June 1, 1979. 54A35.

AMS (MOS) subject classifications (1970). Primary 54F45, 57A10; Secondary 54C55,

Key words and phrases. Cell-like, aspherical, 3-manifolds.

${ }^{1}$ Research supported in part by an NSF grant.

() 1980 American Mathematical Society 0002-9904/80/0000-0106/\$01.50 
that each $n$-dimensional compactum imbeds in a $(n+1)$-dimensional $\mathrm{AR}$ and the result of Sieklucki [Si] that a collection of pairwise disjoint $n$-dimensional closed subsets of an $n$-dimensional ANR is countable can be used to show that the celllike mapping problem for $n$-dimensional compacta is equivalent to the cell-like mapping problem for $(n+1)$-dimensional ANR's. Second, using techniques from [Ed-2], R. Daverman has shown that, for $n \geqslant 4$, if there is a cell-like dimension raising mapping on an $n$-manifold, then there is a cell-like dimension raising mapping on an $(n-2)$-dimensional compactum. Starting with a cell-like mapping $f: M^{n} \rightarrow Y(n \geqslant 4)$, Daverman constructs a cell-like mapping $\bar{f}: M^{n}$ $\rightarrow Y$ with $\bar{f}$ one-to-one on an "infinite 1-skeleton" of $M^{n}$; if $\operatorname{dim} Y=\infty$, then there is a compact subset $Y^{\prime} \subseteq Y$ with $\operatorname{dim} Y^{\prime}=\infty$ and with $\operatorname{dim} f^{-1}\left(Y^{\prime}\right) \leqslant n-2$.

The following illustrate that even with substantial additional hypotheses there are currently few results. In [Dy] Dyer showed that if $f: X \rightarrow Y$ is an open mapping with $\operatorname{dim} X=n$ and with each $f^{-1}(y)$ a point or an arc, then $\operatorname{dim} Y=n$ or $n-1$ provided $Y$ is assumed to be finite dimensional. In [Bor] Borsuk pointed out that a consequence of Smale's Vietoris mapping theorem for homotopy [Sm] is that if a space $Y$ is the image of ANR $X$ under a proper map $f: X \rightarrow Y$ with each $f^{-1}(y)$ an AR, then $Y$ is an ANR provided $Y$ is assumed to be finite dimensional. Both Dyer and Borsuk asked whether or not the assumption of finite dimensionality is necessary; these questions remain unsettled.

\section{BIBLIOGRAPHY}

[Ar] S. Armentrout, Monotone decompositions of $E^{3}$, Topology Seminar Wisconsin 1965, Ann. of Math. Studies, vol. 60, Princeton Univ. Press, Princeton, N. J.

[Bi] R. H. Bing, Decompositions of $E^{3}$, Topology of 3-Manifolds and Related Topics, Prentice-Hall, Englewood Cliffs, N. J., 1962, pp. 5-21.

[Bor] K. Borsuk, Theory of retracts, Monografie Mat., Tom. 44, PWN, Warsaw, 1967.

[Bo] $\mathrm{H}$. Bothe, Eine Einbettung $m$-dimensionaler Mengen in einen $(m+1)$-dimensionalen absoluten Retrakt, Fund. Math. 51 (1962), 209-224.

[Dy] E. Dyer, Certain transformations which lower dimension, Ann. of Math. 63 (1956), 15-19.

[Ed-1] R. D. Edwards, $A$ theorem and a question related to cohomological dimension and cell-like maps, Notices Amer. Math. Soc. 25 (1978).

[Ed-2] - Approximating certain cell-like maps by homeomorphisms (preprint).

[H-W] W. Hurewicz and H. Wallman, Dimension theory, Princeton Univ. Press, Princeton, N. J., 1941.

[Koz-1] G. Kozlowski, Mapping theorems for homotopy, Ph.D. Thesis, University of Michigan, Ann Arbor, 1968.

[Koz-2] - Images of ANR's, Trans. Amer. Math. Soc. (to appear).

[Sch] R. Schori, The cell-like mapping problem and hereditarily infinite-dimensional compacta (preprint).

[Si] K. Sieklucki, A generalization of a theorem of $K$. Borsuk concerning the dimension of ANR-sets, Bull. Acad. Polon. Sci. Ser. Sci. Math. Astronom. Phys. 10 (1962), 433436 (also see correction, 12 (1964)).

[Sm] S. Smale, A Vietoris mapping theorem for homotopy, Proc. Amer. Math. Soc. 8 (1957), 604-610. 35830

DEPARTMENT OF MATHEMATICS, AUBURN UNIVERSITY, AUBURN, ALABAMA

DEPARTMENT OF MATHEMATICS, UNIVERSITY OF TENNESSEE, KNOXVILLE, TENNESSEE 37916 (Current address of both authors) 